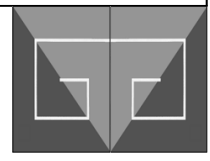

\title{
La RFG monte à la volée
}

\section{Pour une recherche en gestion française qui rende coup pour coup}

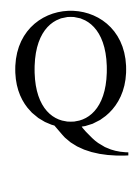

u'il semble loin, ce temps où la RFG était dans les bâches. Condamnée tel un joueur de tennis de seconde zone à ramer au fond du cours pour exister avec peine dans le paysage des revues de management ; renvoyée hors $\mathrm{du}$ top 500 mondial par les classements réalisés par les instances scientifiques de son propre pays. Durant ses trois mandats, Jean-Philippe Denis a remis la RFG au centre du jeu et réussi la métamorphose qu'il avait promise (Denis, 2014). La revue est au plus haut de ce qu'une revue française peut espérer dans le classement FNEGE (rang 2), aux portes du top 100 mondial donc. Elle reçoit près de 400 manuscrits par an, ce qui en fait la revue française de management qui reçoit le plus de soumissions. Les statistiques officielles de consultation fournies par Cairn attestent que ses articles sont lus chaque année par près d'un million et demi de personnes. Audelà du monde scientifique, elle bénéficie d'une audience incontestable auprès du grand public, des étudiants et des professionnels grâce à ses comptes sur les réseaux sociaux (Twitter, LinkedIn, Facebook), et les 200 vidéos tournées dans le cadre du partenariat avec Xerfi canal. 
Vidéos que l'on peut désormais, grâce au travail d'Hugo Gaillard, devenu officiellement depuis le $1^{\text {er }}$ septembre le responsable de la valorisation de la revue, retrouver sur la chaîne youtube de la RFG (https://www.youtube. com/channel/UCW3CGtCIWX6FSLAy1-

$\mathrm{KL} 5 \mathrm{dQ}$ /playlists)

La RFG est revenue au centre du jeu, elle a désormais les deux pieds bien à l'intérieur $\mathrm{du}$ cours, mais nous ne saurions nous en contenter. À l'heure où Amazon diffuse désormais Roland Garros, qu'autour du monde de l'enseignement supérieur et de la recherche règnent les loups du digital, l'heure n'est plus de jouer petit bras. La RFG doit viser la victoire en grand chelem, et pour un français, la seule stratégie qui vaille est d'évidence l'attaque ! Tel le grand Yannick Noah de 1983, la RFG doit dans les prochaines années monter à la volée. Alors que la planète brûle, que malgré d'évidents progrès, les pouvoirs politique, médiatique, intellectuel et économique n'écoutent que peu les chercheurs en gestion, que le grand public persiste à percevoir les gestionnaires comme de méchants cost killers, la RFG doit taper plus fort. Oui, la gestion doit se faire entendre au même titre que l'économie ou la sociologie, et faire en sorte que les savoirs qu'elle produit résonnent aussi forts que les cris de Monica Seles sur un court, tant ces savoirs seront décisifs en vue de faciliter la grande transition environnementale qui est devant nous. Et elle doit le faire bien sûr en Français, langue olympique qui devrait être parlée en 2050 par 750 millions de personnes. Cette langue qui bien que peu utilisée au tennis (ace, smash, etc.), a excusez du peu donné son nom à ce sport, le tennis dérivant comme on le sait du vocable «tenez » qui était prononcé autrefois par le joueur qui sert au jeu de paume.
Valoriser donc les travaux de la communauté gestionnaire francophone, tout en renforçant encore les processus éditoriaux qui permettent à la RFG de fonctionner, et qui sont garants de l'éthique et de l'excellence scientifique qui est sa marque de fabrique depuis bientôt 50 ans. Pour cela, des nouvelles règles de gouvernance (voir ci-après) ont été en juin conçues avec la Fnege et l'éditeur Lavoisier, et validées par le conseil de rédaction. Suite à ces règles, Jérôme Barthélémy, Jean-Noël Kapferer, Alain-Charles Martinet et Bernard Pras, qui ont tant servi la revue et qu'au nom de toute la communauté je tiens à remercier vivement et chaleureusement pour leur engagement et leur soutien, ont quitté le conseil de rédaction le $1^{\mathrm{er}}$ septembre. Ils ont été remplacés par Jean-Philippe Denis (qui en tant qu'ancien rédacteur en chef prend la place de Jérôme Barthélémy), et par Emmanuelle Le Nagard, Linda Rouleau et Anne-Laure Saives. Cette féminisation du conseil de rédaction était une évidence absolue, et permet d'atteindre une mixité essentielle aujourd'hui. Cette mixité qui s'incarne aussi aujourd'hui à la tête de la revue, où j'opère avec Nathalie Dubost dans le cadre d'un double mixte d'ores et déjà complémentaire.

Bref, c'est donc une politique de la terre battue que je vais défendre au sein de la RFG durant mon mandat. Avec le coup droit de Nadal pour rétorquer à ceux qui pensent qu'aucune revue française ne mérite le rang 1, que la RFG est bien plus innovante et intéressante que nombre des revues anglo-saxonnes et aurait toute sa place dans le classement du Financial Times. Avec le revers de Roger Federer pour dire aux professionnels qui rabâchent que les chercheurs en gestion ne savent pas 
leur parler, que s'ils ne peuvent prendre cinq minutes pour lire un papier The Conversation ou visionner une vidéo Xerfi Canal, on ne pourra rien pour eux. Avec la volée de «Martina Navratilova » pour reprendre les journalistes qui nous assimilent à encore des économistes et leur signifier que les sciences de gestion ont une histoire qui leur est propre et ne sortent pas de la cuisse de l'économie (Hatchuel, 2019). Et avec la détente verticale de Yannick Noah pour aller smasher la fusée de Jeff Bezos et la faire illico presto atterrir (Latour, 2017), afin que notre planète ne devienne pas bleue comme une orange !

\section{BIBLIOGRAPHIE}

Denis J.-P. (2014). « La RFG à l'heure de la métamorphose. "Que nul ne soit (plus) censé ignorer la recherche en management..." ", Revue française de gestion, vol. 40, n 238, p. 5-9.

Hatchuel A. (2019). " Exit to the past and voice for the future. Sciences de gestion, sciences fondamentales de l'action collective », Revue française de gestion, vol. 45, $\mathrm{n}^{\circ}$ 285, p. 43-57. Latour B. (2017). Où atterrir? Comment s' orienter en politique, La Découverte. 


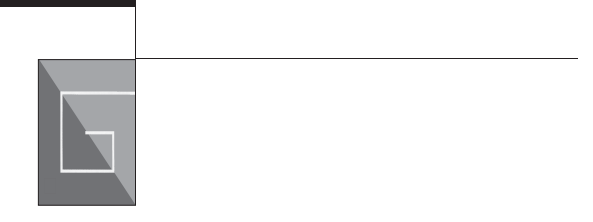

\section{Candidature au poste de Rédacteur en chef de la Revue française de gestion}

Chers membres du conseil de rédaction, Je vous écris pour vous adresser ma candidature au poste de Rédacteur en chef de la Revue Française de Gestion (RFG) et vous faire part de mon désir de succéder au Professeur Jean-Philippe Denis en septembre 2021.

Ma candidature est motivée tout d'abord par mon attachement à la RFG, revue que j'ai découverte alors que j'étais en thèse. Plusieurs des articles de la revue m'ont fortement influencé dès cette période de formation (citons l'article de Jean-Claude Moisdon de 1984 sur la « recherche en gestion et l'intervention », celui d'Armand Hatchuel de 1994 sur les « apprentissages collectifs et les activités de conception », de Philippe Lorino de 1995 sur le « déploiement de la valeur par les processus », etc.). C'est ensuite dans la RFG que j'ai publié l'un des principaux résultats de ma thèse sur les standards logistiques (Rouquet, 2012). De manière naturelle, mon engagement envers la revue s'est au fil des ans affermi. J'ai eu l'opportunité de coordonner un numéro spécial en 2013 sur la thématique du « client acteur de l'organisation » avec deux collègues en marketing (Kiane Goudarzi et Fanny
Reniou). Je suis à partir de cette date devenu un évaluateur régulier de la $\mathrm{RFG}$, et j'ai réalisé depuis une quinzaine d'évaluations, dont une dizaine depuis 2018, et ce en respectant toujours les délais impartis. J'y ai récemment publié avec Valentina Carbone et Christine Roussat deux résultats issus de nos recherches sur les liens entre logistique et économie collaborative $(2016,2018)$ au sein de deux numéros spéciaux (l'un dédié à la « foule », l'autre au « supply chain management »). J'y ai aussi fait paraitre dans le numéro spécial coordonné par Jean-Luc Moriceau, Hervé Laroche et Rémi Jardat sur la nécessité de « retrouver du sens » dans la recherche un texte plus personnel appelant à la « révolte » contre les effets délétères des logiques de ranking. Actuellement, je coordonne enfin le numéro spécial « Ce que la fiction fait aux organisations » avec Caroline Julliot et Marc Lenglet, respectivement spécialistes de littérature et finance, pour lequel nous avons reçu plus de 20 propositions.

À bien des égards, mon histoire personnelle avec la RFG illustre parfaitement la posture scientifique qui est la mienne. Sur le plan disciplinaire, elle témoigne que je suis 
chercheur en logistique, une discipline à laquelle j'ai été formé au CRET-LOG et à laquelle je suis profondément attaché. Mais elle montre aussi que cet attachement n'a jamais été pour moi une prison ! Fidèle à l'idée que la logistique est une discipline d'interface, mes travaux pour la revue se sont ainsi nourris d'un dialogue avec d'autres disciplines de gestion, notamment le marketing et la stratégie. Plus largement, mes liens avec la RFG attestent de mon intérêt pour l'évolution de notre métier d'enseignant-chercheur, ainsi que pour le paysage des sciences humaines et sociales au sens large (sociologie, histoire, économie, littérature, etc.). Sur le plan méthodologique, cette histoire met en évidence que je suis avant tout un chercheur qualitatif. Elle souligne cependant que je ne me cantonne pas à réutiliser la même méthode mais mobilise une diversité d'approches selon les situations : recherche-intervention, études de cas, monographies, analyse netnographique, approche historique, essais conceptuels, etc. Sur le plan académique, elle démontre ma volonté de ne pas rechercher exclusivement l'impact international. Alors que beaucoup considèrent que seule la publication en anglais devrait désormais compter, j'ai, tout en faisant paraître mes travaux dans les meilleures revues anglo-saxonnes de ma discipline, toujours continué à publier en français dans la RFG et d'autres revues françaises. Cet attachement au français me conduit d'ailleurs, contrairement à la plupart de mes collègues, à me revendiquer professeur de « logistique », et non de « supply chain management». Sur le plan de l'impact enfin, cette histoire démontre mon souci de contribuer non seulement à la théorie, mais aussi à la pratique. Au-delà de veiller à écrire mes textes pour la RFG dans une langue qui soit compréhensible au maximum par tout lecteur, j' ai systématiquement produit des versions courtes de mes articles RFG dans différents supports, notamment The Conversation et Xerfi Canal. Ce souci de faire parler la voix de la recherche en gestion dans le débat public m'a conduit dernièrement à intervenir sur les enjeux logistiques de la campagne vaccinale dans de nombreux médias grand public ( $L e$ Monde, Europe 1, LCI, RTL, TF1, etc.). Ce positionnement personnel me semble tout à fait correspondre au positionnement historique de la RFG et avec les évolutions récentes observables au cours des mandats de Jérôme Barthélémy puis Jean-Philippe Denis. Fondée en 1975 à l'initiative de la Fnege, institution clef dans l'essor des sciences de gestion, la RFG est la plus ancienne de nos revues scientifiques ${ }^{1}$. Elle occupe depuis l'origine un positionnement généraliste, et constitue un lieu important et vital de dialogue entre toutes les disciplines. Elle a accueilli au fil du temps les contributions de chercheurs de toutes les disciplines de la gestion, et les derniers numéros spéciaux impulsés par la revue témoignent de l'importance de cette posture généraliste et interdisciplinaire à laquelle je suis très sensible. Par ailleurs, la volonté de la revue a toujours été de ne pas s'adresser aux seuls chercheurs, mais d'être aussi un instrument de dialogue avec les praticiens. Elle publie dans ce cadre une majorité de travaux s'appuyant sur des démarches

1. Si l'on excepte la revue Direction et Gestion des Entreprises, fondée en 1965, et qui a depuis changé de nom et s'intitule la Revue des Sciences de Gestion. 
qualitatives et dont les sections méthodologiques peuvent être appréhendées par un large public. Contrairement à la plupart des revues anglo-saxonnes (et françaises), elle laisse les auteurs libres de structurer leur texte sans imposer le modèle standard anglais (revue de littérature/méthodologie/ résultats/discussion). Pour que les textes soient lisibles, elle requiert des auteurs de résumer leur méthode dans un simple encadré, et de limiter le nombre de références bibliographiques. Elle insiste naturellement pour que les auteurs incluent une partie sur les recommandations managériales, et cherche, à travers sa politique de numéros spéciaux, à être en prise avec l'actualité (dossier spécial sur la Covid-19, la souveraineté, etc.). Cette volonté de ne pas s'adresser aux seuls chercheurs et de nourrir le dialogue chercheurs-praticiens s'est accélérée au cours de la dernière mandature, à travers la forte présence de la revue sur les différents réseaux sociaux, l'organisation des vitrines RFG, l'établissement de partenariats avec The Conversation ainsi qu'avec Xerfi Canal (émission Fenêtres Ouvertes sur la Gestion puis IQSOG). Soulignons enfin que tout cela s'est fait sans renier l'objectif de développer des contributions théoriques majeures !

Dans un contexte récent marqué par l'essor de la logique des rankings, les mandats exercés par Jérôme Barthélémy puis JeanPhilippe Denis ont permis de réaffirmer la place unique et privilégiée de la revue dans le paysage français. Premièrement, alors que la revue avait initialement été sous classée par le CNRS comme par la Fnege, ce qui, dans le contexte actuel de primat des rankings faisait courir un péril stratégique majeur en risquant d'assécher le nombre de soumissions à une revue jugée par les listes moins bonne, ceux-ci sont parvenus à refaire classer au plus haut niveau possible pour une revue française la RFG par la Fnege, et à la faire remonter dans la liste CNRS de rang 4 à $3^{2}$. Cela permet à la RFG de recevoir un volume beaucoup plus important de manuscrits qu'il y a quelques années (400 environ annuellement), et d'être sans doute aujourd'hui la revue francophone qui en reçoit chaque année le plus $^{3}$. Deuxièmement, alors qu'il existe pléthore de revues et qu'il devient difficile d'affirmer une posture singulière dans ce contexte, ils ont contribué à conserver et développer le positionnement original de la RFG. Celle-ci n'est pas comme la majeure partie des revues à destination des seuls académiques, et elle n'est pas non plus vraiment une revue écrite par des académiques pour les professionnels : elle essaye au fond d'être les deux «en même temps ». Telle que je la perçois, la RFG se veut ainsi la revue francophone généraliste de la gestion, qui publie des articles de recherche écrits dans un langage scientifique mais devant être compris par le plus grand nombre. Enfin, les deux dernières mandatures ont contribué à professionnaliser la revue. Cela s'est traduit par une amélioration significative de la rigueur et de la transparence du processus de sélection des articles (qualité des évaluations, délais courts de révision, fréquence de parution, respect des échéances), et par le développement avec le conseil de rédaction de procédures collectives et claires afin d'attribuer les numéros spéciaux.

2. La RFG n'a pu obtenir un 2 mérité, le CNRS ayant mis fin au principe de son classement fin 2020.

3. Les statistiques à ce sujet manquent toutefois. 
Les succès que l'on peut mettre au crédit des derniers rédacteurs en chef ne doivent pas nous faire oublier qu'il ne faut en aucun cas se reposer sur ses lauriers passés dans un monde hypermoderne marqué par le « red queen effect». D'autant que plusieurs évolutions en cours risquent à terme d'avoir des conséquences importantes sur la revue : l'essor porté par le CNRS de l'open access et les bouleversements du paysage de l'édition scientifique ; la montée en puissance de l'idée qu'il faut désormais évaluer la recherche sur la base de l'impact factor à court terme des articles, qui risque de marginaliser encore plus les revues françaises qui bénéficient par définition d'une audience moins importante que les revues de langue anglaise ; la fusion qui tend de plus en plus à s'opérer entre les métiers de chercheur, de journaliste, d'essayiste, et qui invite à questionner la manière dont les chercheurs en sciences de gestion peuvent faire entendre leur voix propre ; l'accélération de la temporalité, qui fait que le temps médiatique et de la publication devient de moins en moins compatible avec le temps long de la recherche ; l'hyperspécialisation disciplinaire à l'œuvre en gestion, qui pourrait conduire au repli de chaque discipline sur soi, voire à une alliance de ces mêmes disciplines contre la posture généraliste ; le développement d'outils de traduction puissants, qui vont amener à reconsidérer la question de la langue dans les travaux, et représenteront des opportunités pour faire valoir internationalement la RFG, etc.

S'il n'est pas possible ici de donner mon point de vue sur l'ensemble de ces aspects, et que chacun de ces sujets doit être réfléchi avec le conseil de rédaction, il me semble cependant que trois directions doivent au moins être suivies dans les prochaines années. Ce sont en tous les cas celles que j'aimerais porter si le conseil de rédaction m'accorde sa confiance. La première est le renforcement du positionnement francophone de la RFG. À l'heure où de nombreuses revues françaises acceptent des articles écrits en anglais, il me paraît qu'une telle voie représente pour la RFG une impasse et le risque d'une perte de singularité. Ne pas suivre cette voie ne signifie cependant pas que la revue ne doive pas chercher à peser sur le plan international. La stratégie à suivre me semble de renforcer ce positionnement francophone comme a déjà commencé à le faire l'actuel rédacteur en chef. La RFG doit ainsi devenir le support privilégié de tous ceux qui cherchent à publier en français, qu'ils soient en France, au Canada, en Afrique ou ailleurs. Alors que la revue reste sans doute encore trop franco-française, des numéros spéciaux pourraient permettre de développer cet aspect (à l'instar de celui sur les recherches africaines), des partenariats pourraient être noués avec des institutions œuvrant pour la francophonie, etc. Parallèlement, des subventions pourraient être recherchées pour éditer des éditions anglaises des textes français de la RFG, en vue de mettre à disposition du plus grand nombre la pensée française en management. Pour cela, pourraient être traduits non seulement une sélection d'articles qui paraissent chaque année, mais aussi certains «classiques » publiés dans le passé par la revue. La deuxième orientation que je chercherai à privilégier est celle de la médiatisation. Bien que des progrès immenses aient été faits sur ce plan, les chercheurs en gestion restent très largement sous-exposés par rapport aux économistes et aux sociologues. Alors que 
la présence médiatique de la RFG s'est affirmée durant les dernières années et fait de celle-ci la revue de gestion la plus visible, il est important de poursuivre cette démarche et de médiatiser encore plus la revue et les chercheurs en gestion. Une stratégie qui pourrait être explorée est notamment celle des liens avec les radios et la presse grand public. Des accords pourraient ainsi être trouvés en vue de produire des chroniques hebdomadaires s'appuyant sur les travaux publiés dans la RFG. Au-delà, une réflexion pourrait être menée afin de créer une rubrique permettant la publication en quelques semaines d'articles courts écrits par des chercheurs. Ces textes courts, qui pourraient être mis en accès libre afin de les diffuser largement, auraient pour objectif de faire valoir, sur des sujets qui agitent médiatiquement nos sociétés, la parole de la recherche en gestion. Enfin, la troisième orientation est celle de la structuration. Alors que la revue reçoit toujours plus de soumissions, et que les chantiers à traiter sont de plus en plus nombreux, il s'agit clairement de jouer plus collectif. Une telle évolution a d'ores et déjà été suivie à travers la création récente du poste de rédacteur en chef adjoint, confié à Nathalie Dubost. Le tandem rédaction en chef-rédaction adjointe doit à mon sens chercher à professionnaliser encore plus la revue dans sa relation aux auteurs. Notamment, il me paraît important de produire désormais des lettres de synthèse des évaluations des articles, qui n'existent pas à notre connaissance actuellement, comme nous en produisons actuellement avec Caroline Julliot et Marc Lenglet pour le numéro spécial sur la fiction. Plus largement, une réflexion sur l'intégration de nouveaux acteurs dans l'équipe pourrait également être conduite, en vue de déléguer par exemple l'éventuelle nouvelle rubrique évoquée plus haut.

Je vous remercie par avance pour l'attention que vous accorderez à ma candidature et me tiens à votre disposition pour toute question. Bien cordialement,

Aurélien Rouquet Paris, le 7 avril 2021 


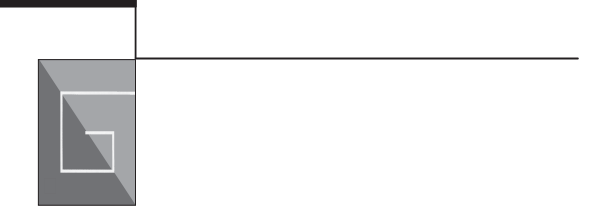

\section{Règles de gouvernance de la Revue française de gestion}

L'équipe de rédaction de la Revue française de gestion est composée au minimum d'un rédacteur en chef, et d'un secrétaire de rédaction. Le rédacteur en chef est élu par le conseil de rédaction, pour un mandat de 3 ans, renouvelable une fois. Son rôle est de diriger la revue, en s'appuyant sur le conseil de rédaction et l'équipe éditoriale.

Le conseil de rédaction accompagne l'équipe de rédaction dans les décisions éditoriales. Il donne son avis sur les propositions de numéros spéciaux faites à la rédaction en chef. Il s'assure de la qualité scientifique des processus éditoriaux. Il veille à ce que les décisions prises respectent l'éthique et l'intégrité scientifique. Il organise l'élection du rédacteur en chef de la revue et participe aux décisions d'évolution au sein de l'équipe éditoriale. Il se réunit au moins une fois tous les trimestres. Il comprend entre 10 et 16 membres.

Les membres du conseil ont un mandat de 3 ans, renouvelable deux fois. La moitié des membres est nommé par la Fnege, l'autre par l'éditeur de la revue Lavoisier, sur la base de propositions faites par le conseil de rédaction. Afin d'assurer la continuité éditoriale, le rédacteur en chef sortant de la revue peut intégrer le conseil pour une durée de trois ans (non renouvelable). Le conseil de rédaction est à l'image de la communauté académique francophone en sciences de gestion, et intègre toutes ses composantes dans leur diversité. Il comprend des membres de différentes institutions, de divers pays, de plusieurs disciplines, et respecte la mixité femmes/hommes. L'acceptation de la qualité de membre du conseil de rédaction suppose un engagement personnel de participer régulièrement aux conseils de rédaction, de contribuer de façon soutenue aux processus de révision, de jouer le rôle d'ambassadeur de la revue auprès des communautés académique et professionnelle en sciences de gestion et de respecter les critères d'éthique et d'intégrité scientifique en usage au sein de la communauté académique. Le conseil de rédaction s'appuie sur un conseil de membres invités, nommés par le conseil de rédaction. Ce conseil regroupe des parties prenantes concernées par la stratégie partenariale de la Revue française de gestion. Le délégué général de la Fnege et l'éditeur de la revue sont membres permanents de ce conseil. Le conseil de membres invités se réunit une fois par an au minimum en vue d'échanger avec toutes les parties impliquées sur les grandes orientations stratégiques de la revue.

Fait à Cachan, le $1^{\text {er }}$ septembre 2021 


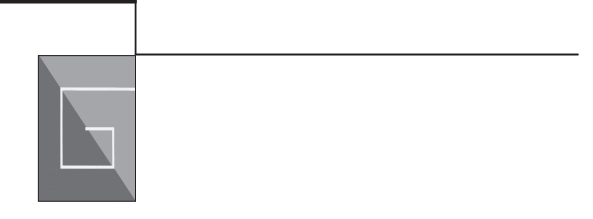

\section{Charte éditoriale de la Revue française de gestion}

Originalité et authenticité des textes. Les auteurs qui soumettent un texte à la Revue française de gestion (RFG) s'engagent à ce que leur texte n'ait pas fait auparavant l'objet d'une publication, et ne soit pas soumis en parallèle à d'autres revues. Ils s'engagent aussi sur le fait qu'ils sont bien les auteurs du texte et que celui-ci est bien un article original. En cas de soupçon de plagiat, l'équipe éditoriale de la RFG peut soumettre l'article à un logiciel anti-plagiat, ou saisir la commission anti-plagiat de la Fnege.

Rejet de la rédaction. Les articles soumis à la RFG via la plateforme Fontis ${ }^{1}$ font d'abord l'objet d'une lecture par un ou plusieurs membres de l'équipe de rédaction. Suite à cette première lecture, l'équipe de rédaction peut rejeter l'article pour deux raisons principales : - l'article n'entre pas dans la ligne éditoriale de la RFG (article s'inscrivant dans une autre discipline que la gestion, ayant une portée trop technique, relevant purement d'une sousdiscipline de la gestion, présentant insuffisamment d'enjeux managériaux, etc.) ;

- l'article est jugé de qualité scientifique insuffisante (écriture médiocre, littérature inadaptée, méthodologie inappropriée, contribution théorique, managériale et/ou sociétale faible, etc.).
Évaluation des articles. Lorsqu'un article n'est pas rejeté, il est affecté à un membre de l'équipe éditoriale, qui se charge de piloter le processus d'évaluation. Celui-ci sollicite pour cela deux ou trois évaluateurs, qu'il sélectionne parce qu'il les juge compétents pour évaluer l'article. Les évaluateurs sont choisis en vue d'éviter au maximum les conflits d'intérêt (un auteur et un évaluateur issus d'un même laboratoire, qui ont par le passé été coauteurs, etc.). L'évaluation est anonyme, et ni les auteurs de l'article, ni les évaluateurs ne connaissent leur identité. Si l'un des évaluateurs pense qu'il existe potentiellement un conflit d'intérêt qui n'a pas été repéré par l'équipe éditoriale, il est de son devoir de le signaler à l'équipe de rédaction.

Processus d'évaluation. À l'issue du premier tour d'évaluation, en fonction du retour des évaluateurs, l'article peut être rejeté, ou bien des demandes de modifications majeures ou mineures peuvent être demandées aux auteurs. Si à l'issue du troisième tour de révision, il existe encore des divergences de vues importantes entre les évaluateurs de l'article (ex. un évaluateur qui accepte, et un autre qui refuse l'article), l'équipe éditoriale sollicite un arbitrage auprès d'un membre du conseil de rédaction ou d'un membre du 
comité de lecture. En fonction de l'avis de l'arbitre, une décision éditoriale est alors prise par l'équipe de rédaction et signifiée aux auteurs.

Durée de l'évaluation. La RFG met tout en œuvre afin qu'une décision éditoriale sur un article soit prise au plus tard un an après la soumission de l'article. Cela suppose que les évaluateurs fassent un retour rapide (idéalement sous 4 semaines), et que les auteurs ne prennent pas trop de temps pour intégrer les modifications qui leurs sont demandées (idéalement 6 semaines).

Mention des auteurs. La liste des auteurs se limite à ceux qui ont apporté une contribution significative au texte. Tous les auteurs doivent être mentionnés, par ordre alphabétique ou selon leur degré d'implication dans l'article (selon leur choix). L'auteur qui est en contact avec la RFG s'assure que tous les coauteurs ont approuvé la version finale du texte qui sera publié.

Dossiers spéciaux. Chaque année la RFG publie plusieurs dossiers spéciaux. Les dossiers spéciaux sont sélectionnés par le conseil de rédaction, sur la base des propositions qui lui sont transmises. Pour avoir une chance d'être sélectionnés, les propositions de dossiers spéciaux doivent être portées par au moins deux auteurs, si possible de deux institutions différentes. Les rédacteurs en chef invités ne peuvent publier d'article dans les dossiers qu'ils coordonnent, mais seulement un article introductif. Pour gérer le dossier spécial, ceuxci utilisent la plateforme de soumission de la revue. C'est aux rédacteurs en chef invités qu'il convient de trouver parmi la base de la revue des évaluateurs afin d'apprécier la qualité des articles, en respectant la politique éditoriale de la RFG. Si le nombre de papiers soumis est trop faible, le dossier spécial peut être annulé. Les rédacteurs en chef invités doivent régulièrement faire le point sur l'avancée du dossier avec l'équipe éditoriale. Ils soumettent pour lecture leur texte introductif à l'équipe éditoriale. Ils participent à la valorisation des dossiers auprès des professionnels et des médias.

Valorisation. Les auteurs qui publient des articles dans la RFG sont incités à valoriser les résultats de leurs travaux auprès des professionnels et du grand public. Ils peuvent le faire par eux-mêmes en relayant leurs articles parus dans la RFG via les réseaux sociaux ou divers médias, ou grâce aux partenariats qui existent entre la revue et certains médias.

Rôle du conseil de rédaction. La RFG s'appuie sur un conseil de rédaction, dont le rôle et le fonctionnement est régi par des règles de gouvernance. Lorsque des cas non prévus par la charte éditoriale sont rencontrés, l'équipe éditoriale fait appel au conseil qui, sur la base des éléments apportés par l'équipe éditoriale, prend alors une décision qui fait jurisprudence pour la suite.

Éthique. Sur le plan éthique, la RFG s'appuie sur la charte éthique commune aux différentes revues éditées par Lavoisier. Les différentes règles en vigueur sont consultables en ligne ${ }^{2}$.

Fait à Cachan, le $1^{\text {er }}$ septembre 2021

2. https://archives-rfg.revuesonline.com/regles_ethiques.jsp 